\title{
FORECASTING TELECOMMUNICATIONS DATA WITH AUTOREGRESSIVE INTEGRATED MOVING AVERAGE MODELS
}

\author{
Nilesh Subhash nalawade ${ }^{\mathrm{a}}$, Mrs. Meenakshi Pawar ${ }^{\mathrm{b}}$ \\ ${ }^{a}$ SVERI's College of Engineering, Pandharpur. \\ nileshsubhash15@gmail.com
}

\begin{abstract}
The ability to perform forecasting calculations is an important thing for forecasting practitioners as it helps them in planning and determining their networks. Accurate forecasting helps forecasting practitioners to provide guidance to business peoples to take important decisions about their business in advance. The International Telecommunication Union (ITU) Recommendation E.507 explains about many techniques for evaluating the forecasting models and choice of the model. But, it does not provide any guidance to select which models are more proper for forecasting the telecommunications series. This paper deals with Autoregressive Integrated Moving Average model for forecasting telecommunication data. Evaluation metrics such as Sum of Squared Regression, Root Mean Square Error, Mean Absolute Deviation, Mean Absolute Percentage Error and Maximum Absolute Error shows that the ARIMA models provides good forecasting performance. Abstract must be of Time New Roman Front of size 10 and must be justified alignment.
\end{abstract}

Keywords - Telecommunication forecasting; ITU Recommendations; ARIMA model; Time series; M-3 Competition data; autocorrelation; Future trend estimation.

\section{INTRODUCTION}

In the growing telecommunications industry the ability to determine the future trends is an important endeavor [12]. The boundaries of the telecommunication industries are increasing very fast and the competition among the industries is also growing as well. Telecommunications companies are in need to develop new strategies to face challenges in providing the best communication services. Many industries depend on data monitoring to improve their business by analyzing the future trends and marketing value for their products. Data forecasting has a major role in network traffic management, optimizing infrastructures and in planning process. $[1,4]$.

The forecasting ability of a time series data is an important thing for the forecasting practitioners. The forecasters can determine the outcome of any event with the priori knowledge about the event. While forecasting telecommunication data, many errors occurs and it motivates to find a better forecasting approach which minimizes the forecasting errors. If a time series data is not forecasted accurately, then reducing the forecasting error will be ineffective. So, it is necessary to find new ways to minimize the effects of poor forecasts [2]. The forecastability of a time series depends on the regularity of the data. If the time series is less regular, then achieving good level of forecasting accuracy is very difficult $[3,9-11,17]$.

There are several techniques for forecasting such as Random walk (simple forecasting method), exponential smoothing methods such as simple exponential smoothing (SES), Holt, Holt-winters, Robust trend etc. In the random walk model, the value of the variable follows a random step for each time interval. It assumes that the current observation is only important and the previous observations provide no information [15]. Simple exponential smoothing is a suitable method for forecasting seasonal data. But it is not a suitable method when there is a trend [16]. Holt method is an extended form of simple exponential smoothing method and it allows forecasting data with trends. It can be improved to deal with both trend and seasonal variations. Holt-winter method overcomes Holt method by considering the seasonal variations [18]. Robust trend methods are suitable for forecasting univariate time series data in the presence of outliers. When using this method, incorrect labeling of samples as outliers may occur.

In this paper, ARIMA model for forecasting telecommunication data is proposed. M3-Copetitive data is used here and the performance is measured in terms of Sum of Squared Regression (SSR), Root Mean Square Error (RMSE), Mean Absolute Deviation (MAD), Mean Absolute Percentage Error (MAPE), Maximum Absolute Error (MAE). The paper is structured as follows. Section 2 describes the M3-Competition telecommunication data. Section 3 describes about the ARIMA based forecast model. Section 4 evaluates forecasting performance. Forecast results are given in section 5 and section 6 concludes the paper. 
International Journal of Engineering Research and General Science Volume 3, Issue 5, September-October, 2015 ISSN 2091-2730

\section{M3-Competition Data}

As the forecasting availability is growing up, there is a need for analyzing the adequacy of forecasting methods. Makridakis and Hibon developed the M-Competition which is considered to be one of the important researches in this field [5, 13]. Data used here are obtained from the Institute of Forecasters. The last edition (M3) is referred to year 2000 and it includes 3003 time series classified in to various types such as micro (828), industry (519), macro (731), finance (308), demographic (413) and other (204) and the different time intervals between the successive observations are gives by yearly, quarterly, monthly and of unknown periodicity('others').

Minimum number of observations is required for each type of data to ensure that enough data can develop an appropriate forecasting model. This minimum was set as 14 observations for yearly series, 16 for quarterly, 48 for monthly and 60 for 'other' series. Table 1 show the classification of the 3003 series according to the two major grouping discussed above. The table shows the number of time series based on both time interval and domain.

Table 1 The classification of the 3003 time series used in the M3-Competition

\begin{tabular}{|c|c|c|c|c|c|c|c|}
\hline Time interval between & \multicolumn{7}{|c|}{ Types of time series data } \\
\cline { 2 - 8 } successive observations & Micro & Industry & Macro & Finance & Demographic & Other & Total \\
\hline Yearly & 146 & 102 & 83 & 58 & 245 & 11 & 645 \\
\hline Quarterly & 204 & 83 & 336 & 76 & 57 & & 756 \\
\hline Monthly & 474 & 334 & 312 & 145 & 111 & 52 & 1428 \\
\hline Other & 4 & & & 29 & & 141 & 174 \\
\hline Total & 828 & 519 & 731 & 308 & 413 & 204 & 3003 \\
\hline
\end{tabular}

\section{ARIMA-based forecast model}

Box and Jenkins introduced the Autoregressive integrated moving average (ARIMA) model. It is used as one of the popular method for forecasting data $[6,14]$. The ARIMA model is used for time series forecasting where the future value of a variable is a linear function of past observations and random errors and is expressed as,

$$
y_{t}=\theta_{0}+\phi_{1} y_{t-1}+\phi_{2} y_{t-2}+\cdots+\phi_{p} y_{t-p}+\varepsilon_{t}-\theta_{1} \varepsilon_{t-1}-\theta_{2} \varepsilon_{t-2}-\cdots-\theta_{q} \varepsilon_{t-q}
$$

Where, $y_{t}$ is the actual value and $\varepsilon_{t}$ is the random error at time $t$, and $\phi_{i}(i=1,2, \ldots, p)$ and $\theta_{j}(j=0,1,2, \ldots, q)$ are model parameters. Integers, $p$ and $q$ are the order of the model. The random errors, $\varepsilon_{t}$ are assumed to be independent and identically distributed with a zero mean a constant variance of $\sigma^{2}$. ARIMA model involves the following three iterative steps [7].

(i) Model Identification: ARIMA model has the assumption that the time series is stationary. So, data transformation is done to produce a stationary time series. For stationary time series, the mean and autocorrelation structure are constant over time. So, differentiation and power transformation are needed to change the time series to be stationary. To identify the appropriate model form, autocorrelation and partial autocorrelation are calculated from the data and it is compared to the theoretical autocorrelation and partial autocorrelation for the various ARIMA models. Steps (ii) and (iii) will determine whether the model is appropriate [8].

(ii) Parameter Estimation: Parameters in ARIMA model is estimated using the nonlinear least square procedure.

(iii) Diagnostic Checking: Several diagnostic statistics and plots such as Histogram, normal probability plot and time sequence plot are used to check the fitness of the model estimated in step (i). Chi-square test can be used to test the model adequacy.

Once a satisfactory model is obtained, the selected model will be used for forecasting.

\section{Evaluation metrics}

Forecast accuracy is measured using Sum of Squared Regression (SSR), Root Mean Square Error (RMSE), Mean Absolute Deviation (MAD), Mean Absolute Percentage Error (MAPE), Maximum Absolute Error (MAE).

(i) SSR: The Sum of Squares Regression (SSR) is the sum of the squared differences between the prediction for each observation and the population mean. 
International Journal of Engineering Research and General Science Volume 3, Issue 5, September-October, 2015 ISSN 2091-2730

$$
S S R=\sum_{l=1}^{L} y-\bar{y}
$$

(ii) MSE: Mean Square Error (MSE) averages the squared prediction error at the same prediction horizon. A derivation of MSE is Root mean Square Error (RMSE).

$$
\operatorname{MSE}(i)=\frac{1}{L} \sum_{l=1}^{L} \Delta^{l}(i)^{2}
$$

(iii) MAD: Mean Absolute Deviation from the sample median (MAD) is the resistant estimator of the dispersion /spread of the prediction error. It is intented to be used when the error plots do not resemble those of a normal distribution.

$$
A D(i)=\frac{1}{n} \sum_{l=1}^{L}\left|\Delta^{l}(i)\right|-M
$$

where, $M=\underset{l}{\operatorname{median}}\left(\Delta^{l}(i)\right)$ and median is the $\frac{n+1}{2}$ th order statistic.

(iv) MAPE: Mean Absolute Percentage Error (MAPE) averages the absolute percentage errors in the predictions of multiple UUTd at the same prediction horizon. Instead of the mean, median can be used to campute Median absolute percentage error (MdAPE) in a similar fashion.

$$
\operatorname{MAPE}(i)=\frac{1}{L} \sum_{l=1}^{L}\left|\frac{100 \Delta^{l}(i)}{r_{\bullet}^{l}(i)}\right|
$$

v) MAE : Mean Absolute Error (MAE) averages the absolute prediction error for multiple UUTs at the same prediction horizon. Using median instead of mean gives absolute error (MdAE).

$$
\operatorname{MAE}(i)=\frac{1}{L} \sum_{l=1}^{L} \mid \Delta^{l}(i)
$$

\section{Results and discussion}

This section presents the performance evaluation of the ARIMA model in M3C data. The ARIMA is implemented using Matlab 8.2.0.701 (R2013b) with a system configuration of 2GB RAM Intel processor and 32 bit OS. To determine the forecasting ability of the ARIMA model, it is applied to the yearly, quarterly, monthly and 'other' data. The Graphical User Interface (GUI) for the ARIMA model for forecasting telecommunication data is shown in figure 1 .

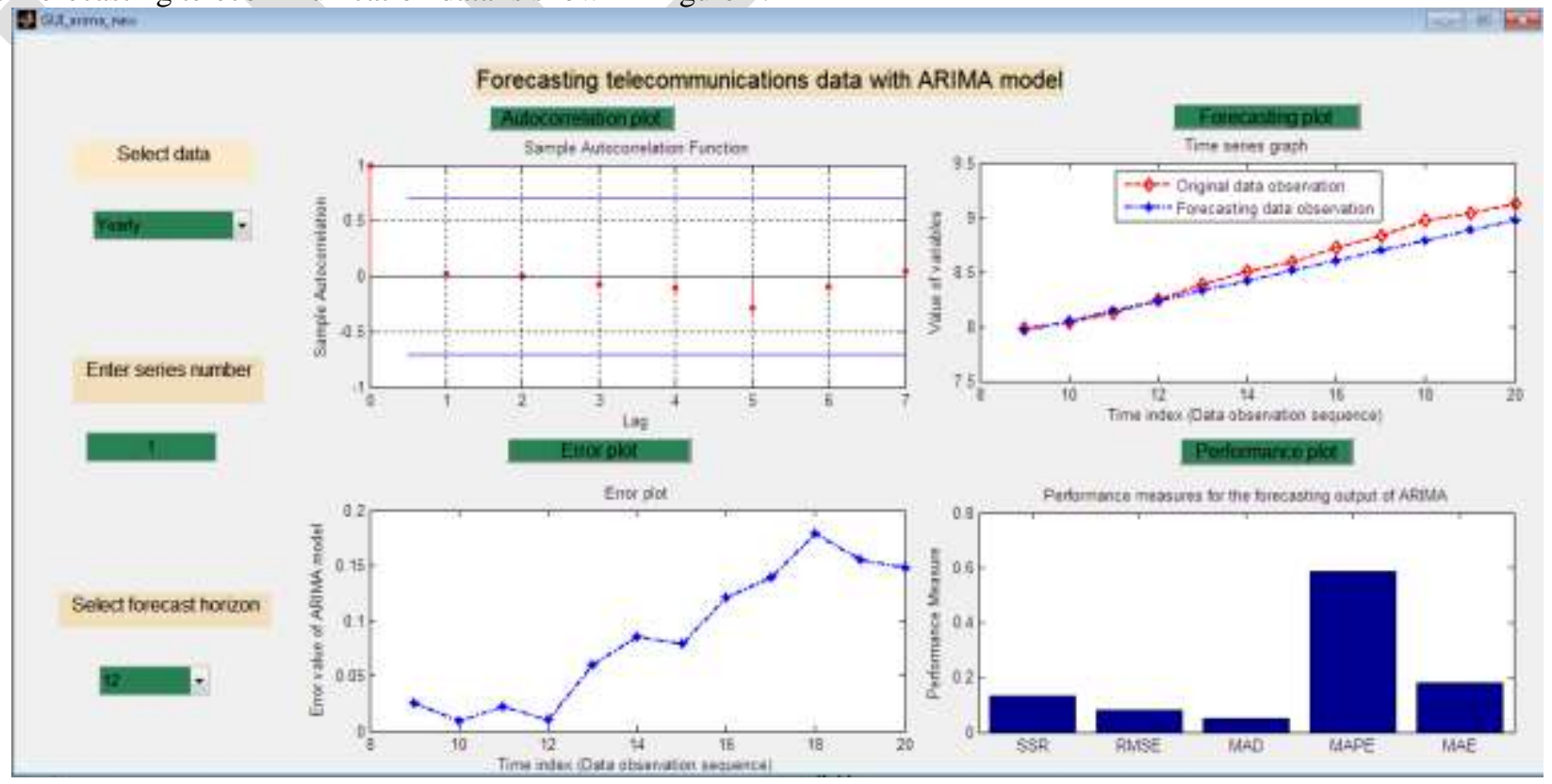


Figure 1. GUI

The autocorrelation plots are used in the model identification stage of the ARIMA time series models. Autocorrelation plots are used to check the randomness in a data set. To find the randomness, the autocorrelations for data values at varying time lags are computed. If the autocorrelation is near to zero for all the time-lag separations, then the data is random. If the autocorrelations are not zero, then the data is not random. Partial autocorrelation are used to find the order of the autoregressive model. The sample autocorrelation plots are given in figure 2. Table 2 shows the statistics of ARIMA $(1,0,0)$ Model extracted from the input data.
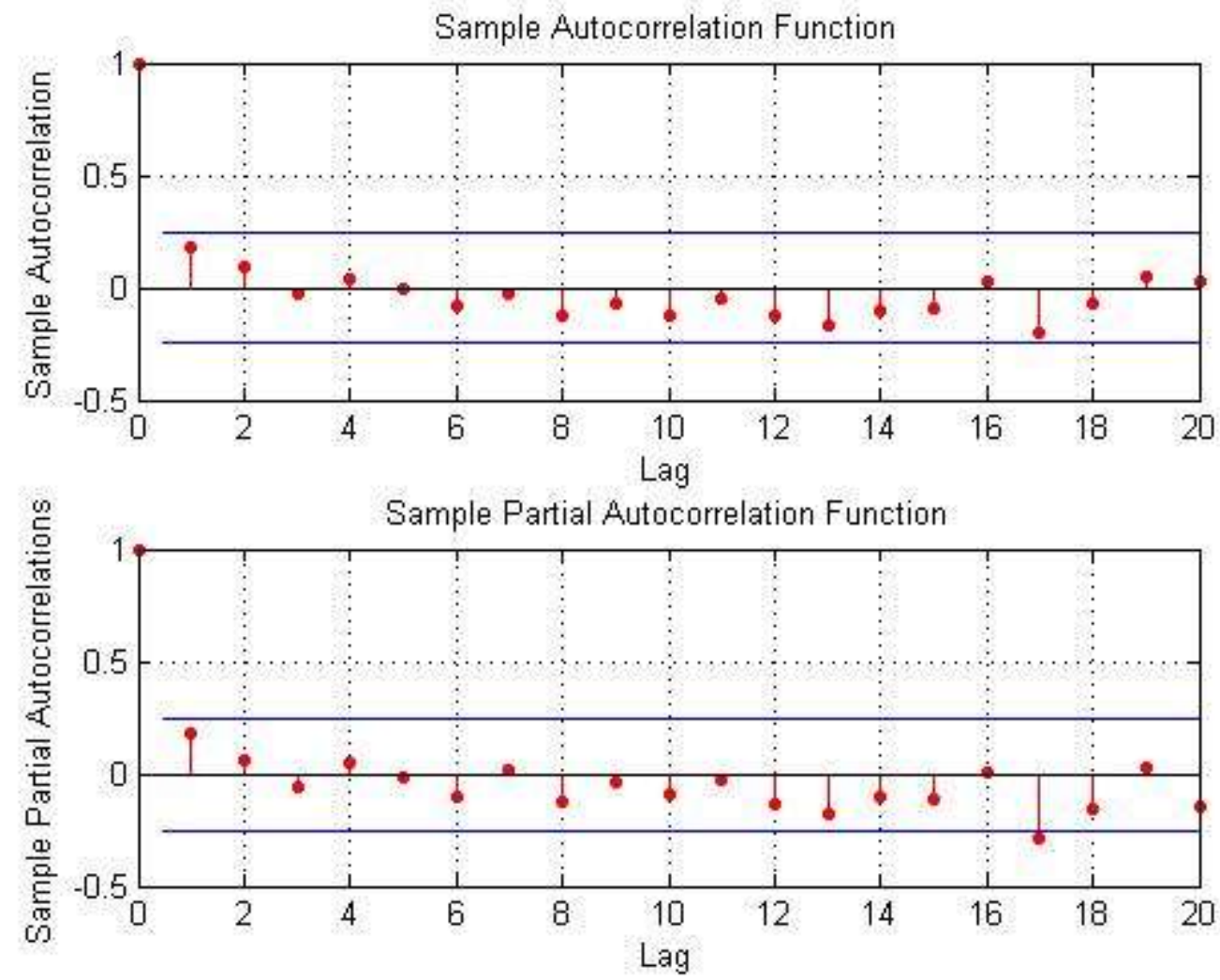

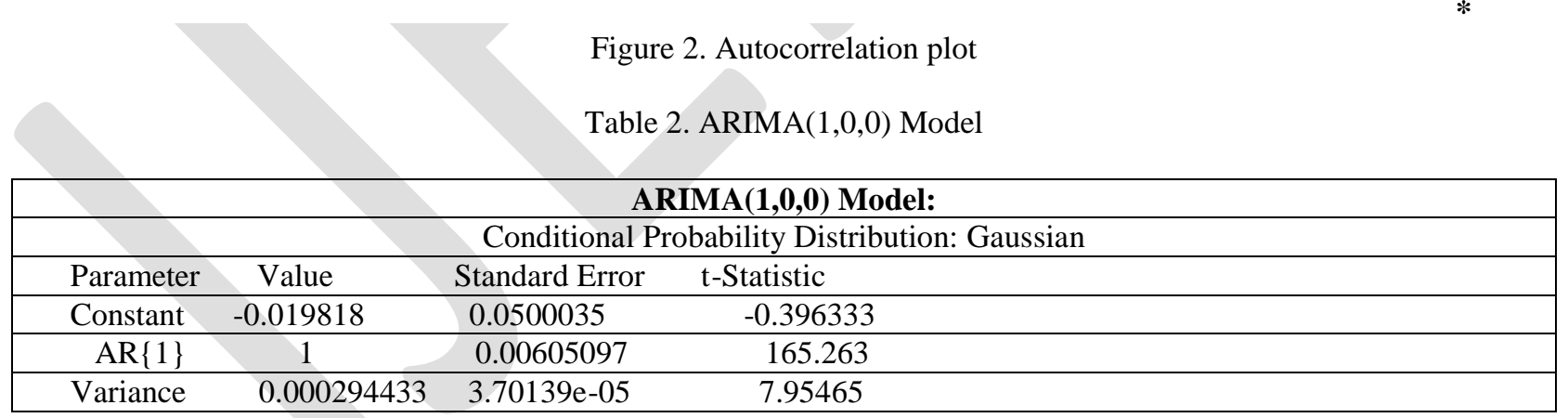

The time-series graph illustrates the data points at successive time intervals. The time is measured on the horizontal axis and the variable is measured on the vertical axis. The time series graph for the comparison between the original data observed and the observed forecasting data and respective performance measure for the yearly data, quarterly data, monthly data and 'other' data are shown in figure 3, 4, 5 and 6 respectively. 

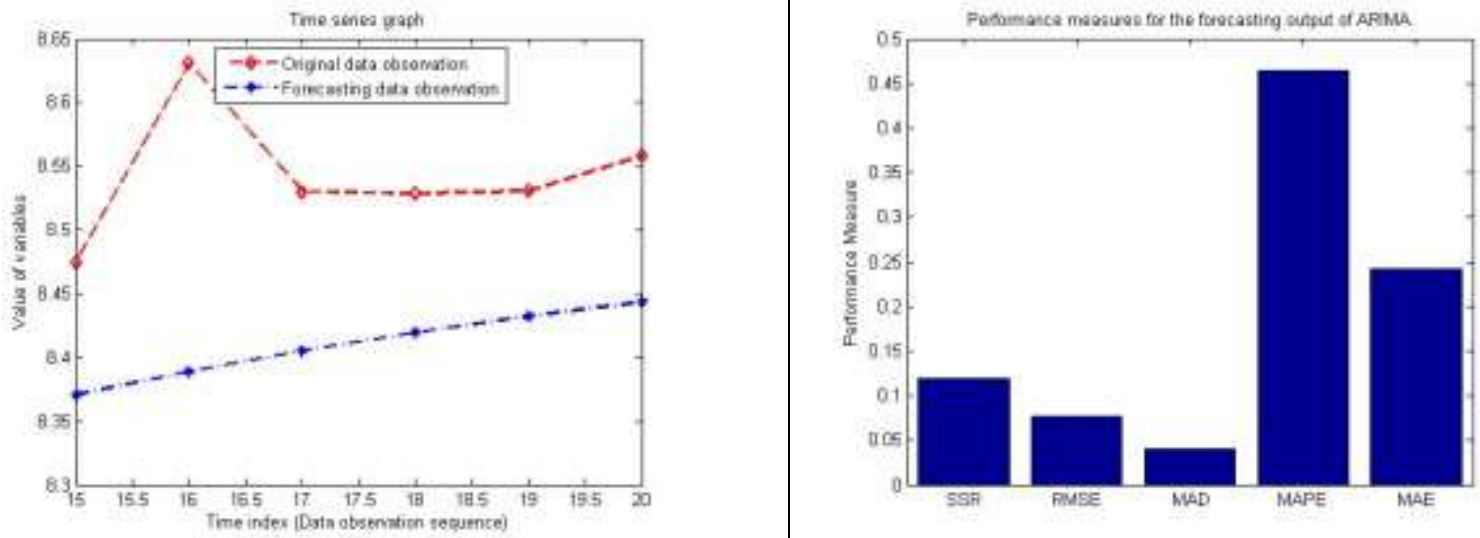

Figure 3. Yearly data

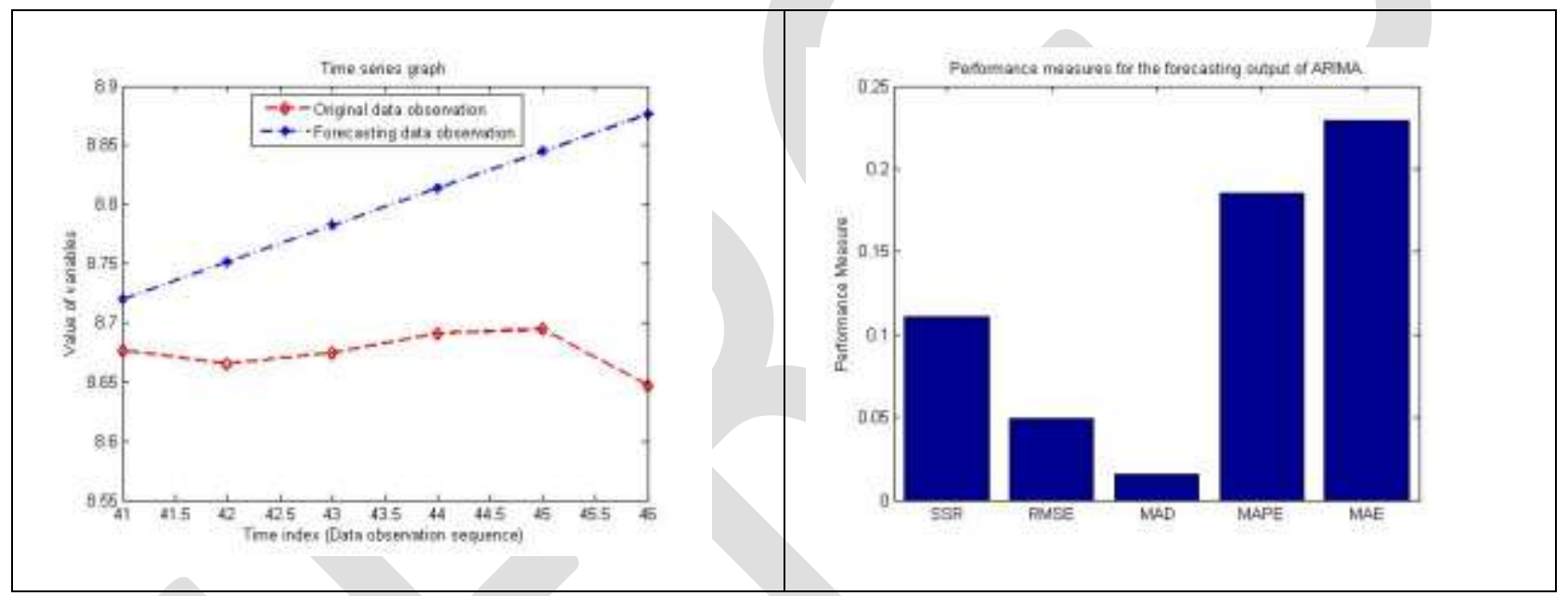

Figure 4. Quarterly data

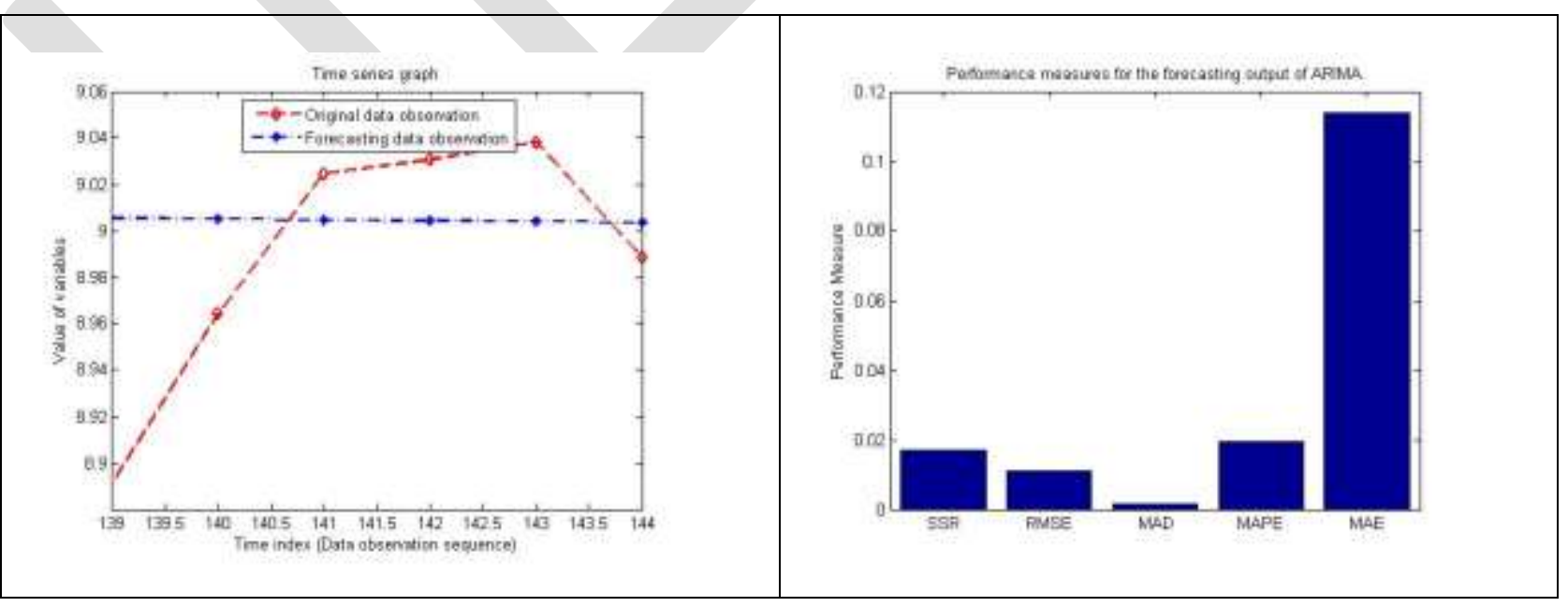

Figure 5. Monthly data 
International Journal of Engineering Research and General Science Volume 3, Issue 5, September-October, 2015 ISSN 2091-2730

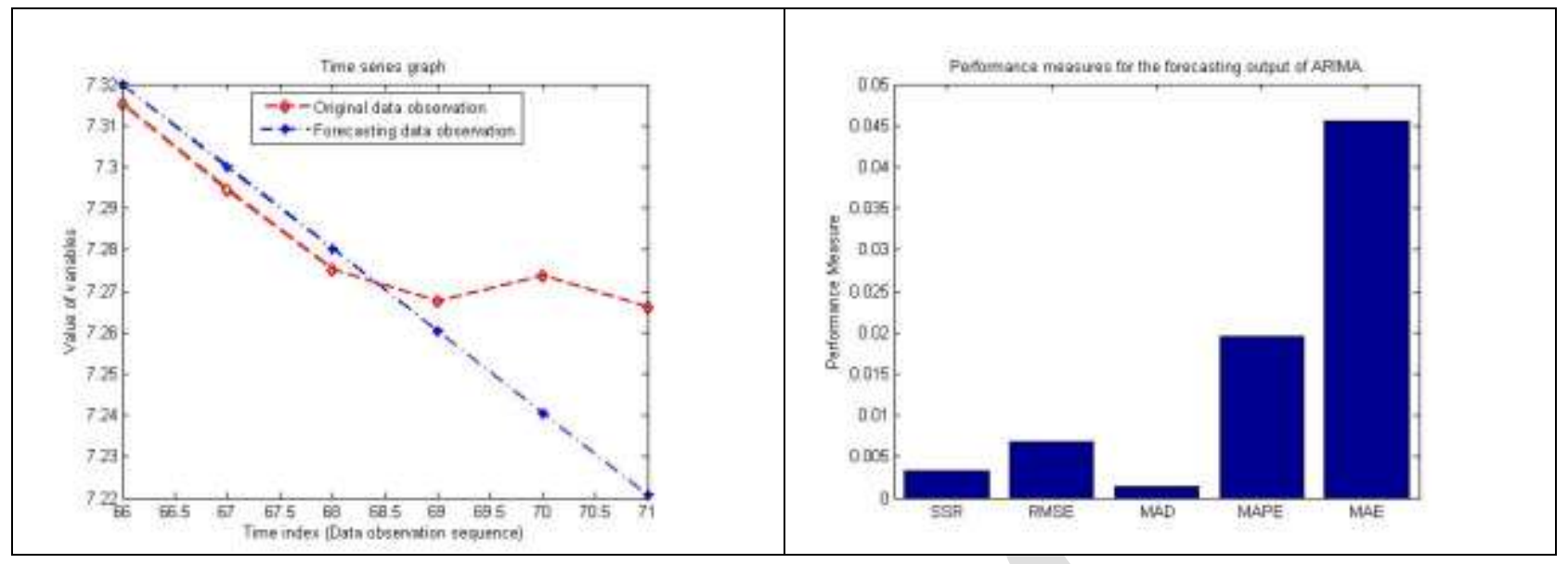

\section{Conclusion}

Figure 6. 'Other' data

In this study, we have applied the ARIMA model to the M3-competition data set of 3003 time series to determine its forecasting ability. It is observed that the ARIMA model provides better forecasting even when little detail about the data is available. The forecasting accuracy of the ARIMA model is measured using parameters such as SSR, RMSE, MAD, MAPE, and MAE for the yearly, quarterly monthly and 'other' data and it is compared with the results given in [19] using MAPE. It showed that the performance of ARIMA model is better than the other models. We therefore conclude that the ARIMA model is the better forecasting method for telecommunication data.

\section{REFERENCES:}

[1] Boylan, J.E. Towards a more precise definition of forecastability. Foresight: the International Journal of Applied Forecasting 2009, 34-40.

[2] Kolassa, S. How to assess forecastability. Foresight: the International Journal of Applied Forecasting 2009, 41-45.

[3] Tashman, L.J. Special feature on forecastability: Preview. Foresight: the International Journal of Applied Forecasting $2009,23$.

[4] Gary Madden, Joachim Tan, "Forecasting telecommunications data with linear models", Telecommunications Policy, vol. 31, pp. 31-44, 2007.

[5] Makridakis, S. and Hibon, M. (2000): "The M-3 Competition: results, conclusions and implications", International Journal of Forecasting, 16, pp. 451-476.

[6] P. F. Pai and C. S. Lin, "A hybrid ARIMA and support vector machines model in stock price forecasting," Omega, vol. 33, no. 6, pp. 497-505, 2005.

[7] G. P. Zhang, "Time series forecasting using a hybrid ARIMA and neural network model," Neurocomputing, vol. 50, pp. 159-175, 2003.

[8] J. E. Hanke and D. W. Wichern, Business Forecasting, Prentice Hall, Englewood Cliffs, NJ, USA, 2009

[9] Armstrong, J., \& Collopy, F. (1992). Error measures for generalizing about forecast methods: Empirical comparisons. International Journal of Forecasting, 8, 69-80.

[10] Fildes, R. (1992). The evaluation of extrapolative forecasting methods. International Journal of Forecasting, 8, 81-98.

[11] Fildes, R., Hibon, M., Makridakis, S., \& Meade, N. (1998). Generalising about univariate forecasting methods: Further empirical evidence. International Journal of Forecasting, 14, 339-358.

[12] Grubesic, T., \& Murray, A. (2005). Geographies of imperfection in telecommunication analysis. Telecommunications Policy, 29, 69-94.

[13] Makridakis, S., Chatfield, C., Hibon, M., Lawrence, M., Mills, T., Ord, K., et al. (1993). The M-2 competition: A real-time judgmentally based forecasting study. International Journal of Forecasting, 9, 5-23.

[14] Parzen, E. (1982). ARARMA models for time series analysis and forecasting. Journal of Forecasting, 1, 67-82.

[15] Simmons, L. F. (1986). M-Competition - A closer look at Nave2 and median APE: a note. International Journal of Forecasting 4, 457-460.

[16] Gardner, E., 2006. Exponential smoothing: The state of the artpart ii. International Journal of Forecasting 22, 637-666.

[17] Kotsialos, A., Papageorgiou, M., Poulimenos, A., 2005. Long-term sales forecasting using Holt-Winters and neural network methods. Journal of Forecasting 24, 353-368.

[18] Fildes, R. \& Petropoulos F. (2013). An evaluation of simple forecasting model selection rules (LUMS Working Paper 2013:2). Lancaster University: The Department of Management Science.

[19] Makridakis, S., \& Hibon, M. (2000).The M-3 competition: results, conclusion and implications. International Journal of

Forecasting, 16, 451-476 